\title{
ENTREVISTA
}

\section{Enseignement de La Sémiologie/Sémiotique Dans Le Monde Actuel com o Professor Sylvano Santini, diretor do Programa de Semiologia da Université du Québec à Montréal}

Licia Soares de Souza

Licia Soares (LS) - Il y a trois programmes de Sémiologie/Sémiotique au monde. L'un est au Canada, à l'UQAM; l'autre est au Brésil, à la PUCUSP ( Pontificale Université de Sao Paulo). Pensez-vous qu'étudier la science des signes actuellement est un atout pour comprendre les entrecroisements de langages de plus en plus nécessaires pour la compréhension du monde?

Sylvano Santini (SS) - Je connais au moins deux autres programmes d'études supérieures en sémiotique dans le monde, à Bologne en Italie et à Tartu en Estonie, sans compter les centres de recherches qui offrent des programmes d'études, comme "Centre for Semiotik » de l'Aarhus University (Danemark) qui offre une maîtrise et le "Center for Cognitive Semiotics » de l'Université de Lund en Suède qui accueille des doctorats et propose des séminaires. Il ne faut pas oublier également que la sémiotique se pratique dans des programmes qui rassemblent les sciences du langage et la communication ou encore des programmes interdisciplinaires en humanités, études culturelles ou cultural studies (dans le monde anglo-saxon). Et finalement, il y a les centres de recherche, comme le CeReS de l'Université de Limoges en France qui a été fondé par Jacques Fontanille en 2002.

Il ne servirait à rien ici de prolonger la liste des centres, associations, instituts, voire revues, qui consacrent une part non négligeable à la sémiotique, car il m'apparaîtrait réducteur, pour ne pas dire inconvenant, de justifier l'utilité de la sémiotique pour comprendre le monde en magnifiant sa pratique sur des données statistiques. Il faut reconnaître toutefois, en tenant compte de la fulgurante ascension de la sémiotique (ou sémiologie) dans les années 1960 et 1970, qu'elle s'est elle-même pris au piège de la popularité en prétendant offrir à aux sciences humaines et sociales le modèle universel d'analyse de la signification tout en ne s'opposant pas à la croyance que sa prolifération dans le milieu académique était le gage de son efficacité. La sémiotique n'a plus cette ambition fort heureusement, et quand bien même 
certains y croiraient encore et la défendraient avec le zèle d'un apostolat, il faut se rendre à l'évidence qu'elle n'en a plus les moyens depuis que la génération qui lui assurait la renommée s'est éteinte.

Ce début de réponse pour le moins inhabituelle a toutefois la vertu de mettre une première chose au clair : il faut abonner l'idée que la sémiotique puisse occuper une position privilégiée pour comprendre le monde. Je me demande bien de toute manière comment on pourrait encore avoir une telle prétention. On doit néanmoins relativiser sa position pour en finir avec le complexe de supériorité qui lui colle à la peau et les consciences qui s'émeuvent ou se troublent à la moindre évocation de son nom. À moins d'avoir l'esprit borner, on n'aura compris que « relativiser » n'a rien à voir ici avec "relativisme ». " Relativiser " signifie, pour moi, penser la sémiotique sans avoir le désir d'en faire un domaine autonome, sans la réduire à l'état de monade scientifique imperméable aux influences de son environnement. « Relativiser » somme toute consiste à s'extraire de la longue mésentente qui a son origine dans une proposition du Cours de linguistique générale qui anticipe l'avenir radieux de la sémiologie en la concevant comme une science qui « a droit à l'existence » et dont la " place est déterminée d'avance ». La sémiologie telle qu'elle se pense et se pratique aujourd'hui ne cherche ni à reconnaître ce droit ni à déterminer cette place. Le vieux rêve disciplinaire l'a maintenue trop longtemps, surtout en Europe et particulièrement en France, sous l'impératif de pureté, rempart inévitable contre les méfaits de la contamination. La sémiotique se relativise en faisant fond sur son devenir indisciplinaire. L'un des points aveugle les plus représentatifs de l'ère sémiotique en France a été l'œuvre de Gilles Deleuze et de Félix Guattari. Ces penseurs ne sont pas en reste d'une réception enviable, aux États-Unis tout particulièrement dans la foulée de la French theory. Mais cette reconnaissance à bien des égards tapageuse intensifie le silence qui enveloppe les deux philosophes et accentue l'absence de réception de leurs travaux parmi l'élite sémiologique française. Ce n'est que très récemment que les ténors du milieu, Jean-Marie Klinkenberg et Jacques Fontanille, s'y intéressent, en considérant la sensation autrement que comme de simples passions de papier, en évoquant entre autres la manière dont l'auteur de Proust et les signes l'envisage dans son analyse figurale des tableaux de Francis Bacon réalisée en 1981. L'avenir de la sémiotique est entre deux chaises depuis que le pilier central de l'édifice sémiotique, le langage, s'est 
ENSEIGNEMENT DE LA SÉMIOLOGIE/SÉMIOTIOUE DANS LE MONDE ACTUEL COM 0 PROFESSOR SYLVANO SANTINI, DIRETOR DO PROGRAMA DE SEMIOLOGIA DA UNIVERSITÉ DU QUÉBEC À MONTRÉAL

élimé. La sémiotique repose désormais sur de multiples bases, notamment la phénoménologie et les sciences cognitives. Elle fait ainsi partie du monde qu'elle aide à comprendre en ce qu'elle est elle-même imbriquée dans un réseau d'influences et de contamination qui ne lui permet plus de prétendre trôner au sommet des sciences qui analysent le sens ou la signification.

Pour arriver à une réponse à votre question, il faut également se débarrasser d'un autre lieu commun. On croit à tort, à mon avis, que la situation actuelle dominée par le capitalisme avancé, les pouvoirs du web ou le Big Data complexifie le monde et sa compréhension. L'impression que la complexité du monde entraîne nécessairement une perte de compréhension abouti bien au sentiment non pas tant d'insécurité mais de manque qui éveille le désir de trouver un modèle puissant d'analyse. Or, au lieu d'apaiser ce désir maniaque en identifiant une façon de parler et de penser qui s'avèrent appropriées au sein du milieu académique pour analyser le mystère mondial (un «lieu spécial» dirait Aristote), on doit d'abord reconnaitre que l'impression de complexité du monde ne date pas d'hier et qu'elle semble revenir ponctuellement hanter les esprits lorsqu'elle laisse présager la force indomptable et chaotique de la multitude. Les rencontres entre les cultures ont toujours eu lieu, comme les mélanges et les impuretés ont miné l'ordre centralisé des empires. Iouri Lotman en fait grand cas en présentant son concept de " sémiosphère ". Ce concept peut aider à faire voir à quel point la tentation de pureté du corps de doctrine, un lieu spécial du discours, expliquerait en partie l'étiolement rapide des disciplinaires qui ont succombé à la tentation sectaire, comme l'École de Paris en sémiotique dans les années 1970 (j'ai écrit un article sur cette histoire qui paraîtra à l'automne 2018 dans la revue d'exploration sémiotique $C y$ gne noir). La sémiosphère est un concept qui propose un modèle d'univers de discours qui est analogue à celui naturel du vivant : la biosphère de Vernadsky. L'analogie entre les deux modèles tient sur le fait qu'ils se caractérisent par l'hétérogénéité de leurs éléments qui garantit le dynamisme et l'énergie, en somme la vie, au sein d'un monde qu'il soit naturel ou discursif. Sans hétérogénéité, en somme tout structure vivante ou sémiotique risque l'entropie et la mort. Lotman ajoute toutefois qu'une trop grande hétérogénéité de la sémiosphère, c'est-à-dire une structure dont la diversité de ses éléments et de leurs fonctions la rendent pour ainsi dire infigurable, ferait en sorte qu'elle serait trop confuse pour donner consistance à un univers. C'est pourquoi il propose de concevoir la 
sémiosphère comme une structure asymétrique, en la divisant entre un centre qui a pour fonction d'assurer la cohésion de la structure et une périphérie qui, elle, a pour fonction d'en assurer l'hétérogénéité. Le centre de la sémiosphère se révèle au moment de l'autodescription qui est une réaction à la trop grande hétérogénéité d'un système :

La forme la plus élevée de l'organisation structurelle d'un système sémiotique, ainsi que l'acte qui mène cette organisation à son terme, survient lorsque ce système se décrit lui-même. C'est l'étape où les grammaires sont écrites, les coutumes et les lois codifiées.

L'étape d'autodescription est une réaction nécessaire à la menace d'une trop grande diversité à l'intérieur de la sémiosphère: le système pourrait perdre son unité et son identité, et se désintégrer (Youri Lotman, La sémiosphère, Limoges, Presses Universitaires de Limoges, 1999 [1966], p. 17).

Lotman imagine la structure asymétrique de la sémiosphère sous la forme dialogique: le centre se comporte comme un individu qui parle au « je » et considère la périphérie comme un "vous ». La frontière qui s'établit ainsi entre le moi et l'autre représente bien la forme de vie qu'ont prise dans l'histoire de nombreux épisodes qui ont marqué des périodes troubles, comme les invasions barbares. Si Lotman reconnaît la nécessité de l'étape de l'autodescription, il valorise surtout ce qu'il appelle les « espaces frontaliers » où ont lieu les phénomènes de transferts et de contamination : bilinguisme, métissage, créolisation, etc. En plaidant en faveur des "dialogues incessants ", Lotman révèle sa proximité avec l'esprit dialogique de Bakhtine et reconnaît l'avantage des invasions barbares. En somme la complexité du monde ne doit pas être considéré comme une période mais comme le signe d'une période de décentrement et de création.

Pour répondre maintenant à votre question, la sémiotique n'est pas un " empire dans un empire " pour reprendre ce que Spinoza disait à propos de l'esprit dans le monde; elle n'est pas un lieu privilégier en dehors du monde à partir duquel on le regarderait ou encore elle ne considère pas le monde comme un tableau accroché sur un mur. La sémiotique fait partie du monde complexe qu'elle doit comprendre, et elle ne pourra y arriver qu'en se comprenant elle-même. Et c'est en partie ce que j'ai fait dans les paragraphes qui précèdent en faisait intervenir des concepts sémiotiques. Si ce que ma réponse vous a été d'une quelconque utilité, je pourrais dire alors que la sémiotique peut en effet encore servir à comprendre le monde. 
ENSEIGNEMENT DE LA SÉMIOLOGIE/SÉMIOTIOUE DANS LE MONDE ACTUEL COM 0 PROFESSOR SYLVANO SANTINI, DIRETOR DO PROGRAMA DE SEMIOLOGIA DA UNIVERSITÉ DU QUÉBEC À MONTRÉAL

LS -Dès le temps que j'ai fini mon doctorat en Sémiologie à l'UQAM, on me demande si la profession de sémioticien(enne) est réglementée. Si cette profession existait, en quoi consisterait-elle, d'après vous?

SS-Après la réponse que j'ai formulée à votre première question je serais mal venu de concevoir, ne serait-ce que par un jeu de l'esprit, la sémiotique comme une profession réglementée. Je vous avoue même que j'en serais bien incapable, comme si une impulsion vitale émanant de ma forme de vie m'empêchait d'y répondre.

LS-Vous travaillez sur une conception de " sémiologie tardive ». Pouvez-vous nous en expliquer les bases?

SS- Cette appellation est à l'origine une boutade que j'ai lancée pour m'opposer à la stagnation de la pratique sémiologique des héritiers de l'École de Paris, des "greimassiens » dirait Erik Landowski. Je l'ai considérée plus sérieusement récemment dans l'article intitulé La sémiologie à l'époque tardive : lectures rétrospectives et actualisantes des formes de vie de Greimas et de l'École de Paris qui paraîtra à l'automne 2018 dans la revue d'exploration sémiotique $C y g n e n$ nir $^{1}$, article que j'ai déjà évoqué plus tôt. J'ai répondu déjà en grande partie à votre question plus tôt. Je tiens à ajouter ceci cependant pour que vos lecteurs saisissent bien la dimension historique de l'appellation Sémiologie tardive.

Le marqueur temporel " tardif » signifie une période historique de bouleversement, de changement, de rupture, etc. qui suit une période caractérisée, elle, par un recentrement des intérêts et des manières de faire autour d'un pôle dominant. C'est dans cet esprit que l'envisagent Ernest Mandel dans Le troisième âge du capitalisme (1973) et Fredric Jameson Le Postmodernisme ou la logique culturelle du capitalisme tardif (1984). Le capitalisme tardif est une période complexe où s'enchevêtrent tous les domaines sous les conditions économiques mondiales. Cette période succède à l'ère impériale du capitalisme monopolistique, où la hiérarchie entre les

${ }^{1}$ http://www.revuecygnenoir.org. 
classes et le pouvoir de la bourgeoisie étaient évidents. C'est également dans cet esprit que Fredric Jameson utilise le marqueur "tardif» dans Le Postmodernisme ou la logique culturelle du capitalisme tardif (1984) pour périodiser le modèle culturel du postmodernisme, marqué par une crise de la représentation des sujets individuels dans le système-monde découlant de l'abolition des frontières entre les domaines qui prévalaient dans le modernisme. "Tardif » signifie donc, chez ces deux auteurs, une période qui déstabilise les fondements qui garantissaient la stabilité et la reconnaissance du système dominant dans la période précédente. Si l'Antiquité tardive la considérait comme un moment de déclin ou de décadence en fonction de causes internes ou externes, il n'en reste pas moins que l'aspect chaotique et agité de la période tardive peut être vécu aussi bien sur le mode exaltant du renouvellement que sur celui, sombre, de l'angoisse et de la disparition civilisationnelle (l'ambiguïté des affects à la période tardive a été notée par Jameson également). C'est dans cet esprit que j'utilise le marqueur temporel tardif comme une période où les repères qui assuraient la stabilité d'un système dominant sont contaminés par des données qui lui étaient jusqu'alors étrangères.

Or, après la période centralisatrice des années 1960 à 1980 où l'École de Paris a joué le rôle de pôle dominant, la sémiologie est aujourd'hui à son époque tardive. J'ai résumé l'essentiel de cette époque dans la réponse à votre première question.

LS- Votre mandat de directeur du programme de Sémiologie s'achève. Pouvez-vous nous dire, en quelques mots, les aspects les plus positifs de votre expérience?

SS- Il faut préciser d'emblée que la tâche du directeur du programme de Sémiologie à l'UQAM est essentiellement administrative : présider les séances des différents comités du programme (admissions, organisation de l'offre de séminaires et de l'évaluation des examens doctoraux et des thèses, bourses pour les étudiant.e.s, etc.) et assurer le bon cheminement des étudiant.e.s dans le programme. La tâche à la direction du programme n'a rien d'extraordinaire, et ne comporte pour ainsi dire ni de hauts ni de bas. Je dirais toutefois qu'elle m'a permis de développer des liens plus étroits avec les étudiant.e.s et les collègues. Il faut dire que le programme implique trois 
ENSEIGNEMENT DE LA SÉMIOLOGIE/SÉMIOTIOUE DANS LE MONDE ACTUEL COM 0 PROFESSOR SYLVANO SANTINI, DIRETOR DO PROGRAMA DE SEMIOLOGIA DA UNIVERSITÉ DU QUÉBEC À MONTRÉAL

facultés et cinq départements ${ }^{2}$, et qu'il est par conséquent un lieu privilégié pour rencontrer des chercheur.e.s provenant d'horizons divers mais dont les préoccupations se recoupent autant par les questions qui sont posées aux objets d'étude que par les outils conceptuels pour les analyser. Il y a, pour le dire ainsi, une communauté d'intérêt, pour ne pas dire interprétative, qui prend forme autour des avenues contemporaines qui interrogent la sémiose, la production et les effets de sens. La fonction à la direction permet d'avoir un point de vue plus ou moins rapproché sur toutes les recherches en cours au sein du programme. Je le conçois donc en partie comme un poste d'observation qui permet de relever certaines tendances dans les thèmes et les sujets de recherche et de prendre le pouls de l'intérêt de la jeune génération de chercheur.e.s pour les études sémiotiques qui exigent non seulement une capacité à concevoir et développer des analyses d'œuvres d'un point de vue théorique, mais un réel plaisir à le faire.

Je terminerais en disant que le programme de sémiologie de l'UQAM va connaître une modification majeure. Si tout se passe comme prévu, le programme modifié devrait être officiellement en place à partir de septembre 2019. Le programme s'intitulera désormais " Doctorat interdisciplinaire en études sémiotiques ». Cet intitulé accentue le caractère interdisciplinaire de la sémiotique, ce qui permet mieux faire apparaître la réalité du programme qui est un espace interdisciplinaire pour la recherche et l'enseignement. Le choix d'avoir mis de l'avant le caractère interdisciplinaire s'explique par le fait que la sémiotique n'est pas considérée, dans l'esprit du programme, comme une discipline en soi mais comme un domaine d'études qui se situe au carrefour de plusieurs disciplines. Le contenu des séminaires, les sujets de thèses et les activités de recherches des professeurs et professeures qui participent au programme interpellent l'interdisciplinarité sur plusieurs plans en la questionnant, la pratiquant ou la théorisant. Enfin, ce nouvel intitulé marque l'évolution de la discipline en utilisant le terme " sémiotique » au lieu de "sémiologie» qui, lui, est associé à une conception qui est historiquement datée. Le programme changera également de nature et durée. Contrairement au programme actuel qui peut admettre les étudiant.e.s qui

\footnotetext{
${ }^{2}$ La Faculté des arts, des sciences humaines et de communication, ainsi que les départements d'études littéraires, d'histoire de l'art, de philosophie, de communication sociale et publique et l'École des médias.
} 
ont terminé des études de premier cycle pour un parcours doctoral de 6 ans et de 120 crédits, le programme modifié proposera uniquement un doctorat de 4 ans et de 90 crédits qui n'admettra que les candidat.e.s détenteur.rices d'un diplôme de maîtrise. Ce changement est attribuable à l'augmentation, depuis 30 ans, des programmes de cycles supérieurs à l'UQAM et, plus largement, dans les autres universités québécoises et canadiennes. 